\title{
Interior Void Classification in Liquid Metal using Multi-Frequency Magnetic Induction Tomography with a Machine Learning Approach
}

\author{
Imamul Muttakin, Member, IEEE, and Manuchehr Soleimani
}

\begin{abstract}
Identification of gas bubble, void detection and porosity estimation are important factors in many liquid metal processes. In steel casting, the importance of flow condition and phase distribution in crucial parts, such as submerged entry nozzle (SEN) and mould raises the needs to observe the phenomena. Cross-section of flow shapes can be visualised using the magnetic induction tomography (MIT) technique. However, the inversion procedure in the image reconstruction has either limited resolution or complex computation degrading its real-time capability. Additionally, in some cases, the actual image may not be essential whereas the void fraction or porosity needs to be estimated. This work proposes an interior void classifier based on multi-frequency mutual induction measurements with eutectic alloy GalnSn as a cold liquid metal model contained in a 3D printed plastic miniature of an SEN. The sensors consist of eight coils arranged in a circle encapsulating the column, providing combinatorial detection on conductive surface and depth. The datasets are induced voltage collections of several non-metallic inclusions (NMI) patterns in liquid metal static test and used to train a machine learning model. The model architectures are a fully connected neural network (FCNN) for 1D; and a convolutional neural network (CNN) for 2D data. The classifier using 1D data has been trained to approximately $86 \%$ accuracy on this dataset. CNN classification using multi-dimensional data with more classes produces $96 \%$ of test accuracy. Refined with representative flow scenarios, the trained model could be deployed for an intelligent online control system of the liquid metal process.
\end{abstract}

Index Terms - Classification, liquid metal, machine learning, magnetic induction tomography, non-metallic inclusion.

\section{INTRODUCTION}

$\mathbf{T}$ HE level of void and/or non-metallic inclusions (NMI) needs to be estimated in many liquid metal applications. In continuous steel casting, the presence of NMI, occurring at primary stage and then more crucial at casting process (multiphase flow of molten metal and argon gas from tundish to mould), affects the steel cleanliness. Therefore, it is important to observe liquid metal flow shape and structure [1]. Another application that necessitates the observation of porosity of metal in its liquid phase is foam manufacturing [2]. The porosity of the liquid metal will determine the final solid porous metal. Liquid metal is also used for cooling nuclear reactor. Here the void fraction is important for heat exchange, and was detected using optical fibre sensing system [3].

Magnetic induction tomography (MIT) system produces

Submitted for review on ... March 2021

This project has received funding from the European Union's Horizon 2020 research and innovation programme under the Marie SkłodowskaCurie grant agreement No 764902.

I. Muttakin is with the Department of Electronic \& Electrical Engineering, University of Bath, Claverton Down, BA2 7AY, United Kingdom (email: i.muttakin@bath.ac.uk).

M. Soleimani is with the Department of Electronic \& Electrical Engineering, University of Bath, Claverton Down, BA2 7AY, United Kingdom (e-mail: m.soleimani@bath.ac.uk). mutual induction values as boundary measurements which are then transformed into cross-sectional image of conductivity distribution. This capability is further enriched by employing frequency-sweep on each measurement resulting in spatiospectral information. Structure of conductive body can be explored using the aforementioned spectroscopy [4]. However, spatial resolution is limited especially for recovering small and dispersed interior non-conductive disturbances. Exhaustive algorithm or post-processing are the usual treatments, still they could not satisfy demands from some industrial applications.

The advancement in artificial intelligence (AI) is accelerated by the availability of data generated from potent sectors. Since the algorithm shows its capability for image interpretation, its implementation progressively finds broad area of medical, industrial and informatics [5] [6]. This trend also applies in tomography technology [7] [8], where the data and/or the resulting image can be exploited to obtain conclusive outputs. Machine learning (ML) utilised in tomography research has mainly been for the reconstruction scheme [9] [10], image enhancement [11] [12], and various post-processing mechanisms [13] [14]. Those methods are rarely directly targeting primary information embedded in the measurement data. Bypassing complexity of tomographic image reconstruction, the detection based on raw-data will reduce the computational resources in 
the deployment phase [15]. Moreover, an end-to-end learning of spatio-temporal feature from raw tomography sensor data was assessed to be an efficient scheme [16].

Key parameters in multiphase flow were predicted from multiple sensors reading using a deep-learning method [17]. The model demonstrated generalisation capability for potential field measurement. The work presented in [18] took direct approach to map the relationship between measurements from electrical capacitance tomography (ECT) sensors and hydrodynamics parameters of flow patterns in fluidised bed. Although reconstruction process was still conducted to infer the process parameters, it experimentally collected training samples offline and then the model was used for on-line monitoring to estimate solid concentration and bubble diameter.

Previously, in cold miniaturised static model experiments, we investigate common two-phase liquid-gas flow scenarios such as full-stream, stratified, bubbly, and annular. These basic flow shapes have been reconstructed with conventional MIT [19]. Accordingly, adaptation of AI become more common in the metallurgy sector [20] [21]. Thus, data-driven method and system are prospective for field implementation to extract useful information in helping production. This work attempts to produce classification of flow inner structure based on multifrequency mutual induction measurements data.

\section{Machine Learning Method using Mutual INDUCTION DATA}

Among several techniques in employing ML for classification problems reviewed in [22], the following work trains the network from scratch while adjusting an efficient architecture for the given problem and dataset.

For dense layer, where each input feature is assigned a vector of weights that connects to activation output, this operation applies [23]:

$$
\begin{gathered}
z^{i}=W^{i} x+b^{i} \\
h^{i}=f^{i}\left(z^{i}\right)
\end{gathered}
$$

here, $W^{i}$ and $b^{i}$ are weights and biases at ith layer respectively. The layer's input is $x$, and the resulting linear activation $z^{i}$ is transformed by a non-linear activation function $f^{i}$.

Activation functions are vary such as sigmoid or hyperbolic tangent. In subsequent sections, Rectified Linear Unit (ReLU) will be used.

$$
f(z)=\max (0, z)
$$

On the other hand, convolutional neural network (CNN) conducts:

$$
z_{n}^{i}=K_{n}^{i} * x+b_{n}^{i}
$$

where $n$ is the index of feature map, $K_{n}^{i}$ is the $n t h$ filter kernel, and $*$ is the convolution operator.

In order to reduce the computation cost, CNN layer is usually accompanied with pooling layer for sub-sampling the feature map. Max operation finds the maximum value.
The performance is evaluated using accuracy, i.e. ratio of correctly classified samples vs all available samples [21]:

$$
a c c=\frac{\sum_{m=0}^{k-1} c_{m, m}}{\sum_{m=0}^{k-1} \sum_{n=0}^{k-1} c_{m, n}}
$$

where $c_{m, n}$ are the elements of the confusion matrix. The network is also trained to minimise the loss between prediction and true labels.

Mutual induction measurements are conducted using twoport method with LCR meter and additional switching module (Keysight Technologies) for sequentially selecting a pair out of 8 -coil array at a time. This measurement system has signal-tonoise ratio (SNR) between $60-90 \mathrm{~dB}$, where measurement at low-frequency opposite-coil has the lowest SNR; and highfrequency adjacent-coil has the highest SNR. The detailed configuration was described in [24]. For the following work (see Fig. 1), all 28 (pair-combination) mutual induction coils are measured and swept from $100 \mathrm{~Hz}$ to $100 \mathrm{kHz}$ (logarithmic scale with 28 points).

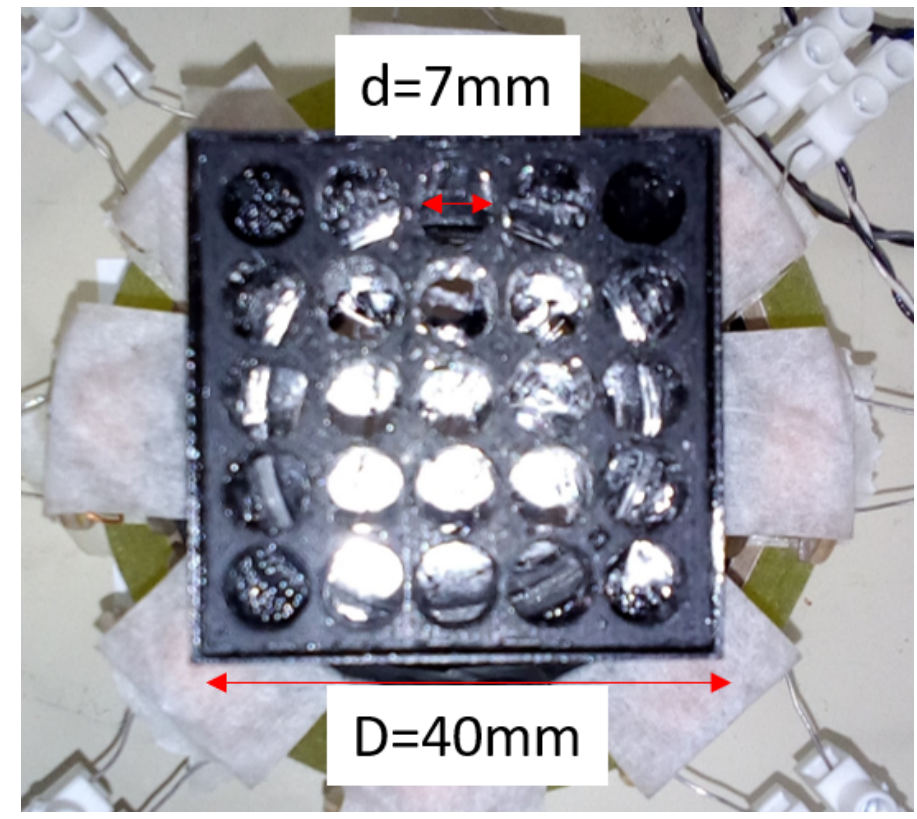

Fig. 1: Liquid metal column with grid for wood inclusion

Pixelated data vector was commonly used as either main or additional input for improving traditional image classification performances [25]. This form of pseudo-image can be built from array of sensors' reading on the first axis and another measurement dimension on the second axis. Example of classifier model which is built on limited training images was reported by [26]. Nevertheless, this dataset will be a valuable framework in developing tomographic sensing interpretation using ML [27] [28].

Two methods are investigated: traditional dense fully connected neural network (FCNN) for classifier using onedimensional (1D) data, and CNN for classifier using twodimensional (2D) data. Both are implemented in Keras 2.4.0 framework [29] with TensorFlow 2.3.0 backend [30]. 


\section{FCNN CLASSIFIER}

In FCNN classifier, the data are built from phase-difference (object against reference full condition). For each frequency, norm function is applied to 28 mutual induction values, hence resulting in normalised one-dimensional dataset shown in Fig. 2.

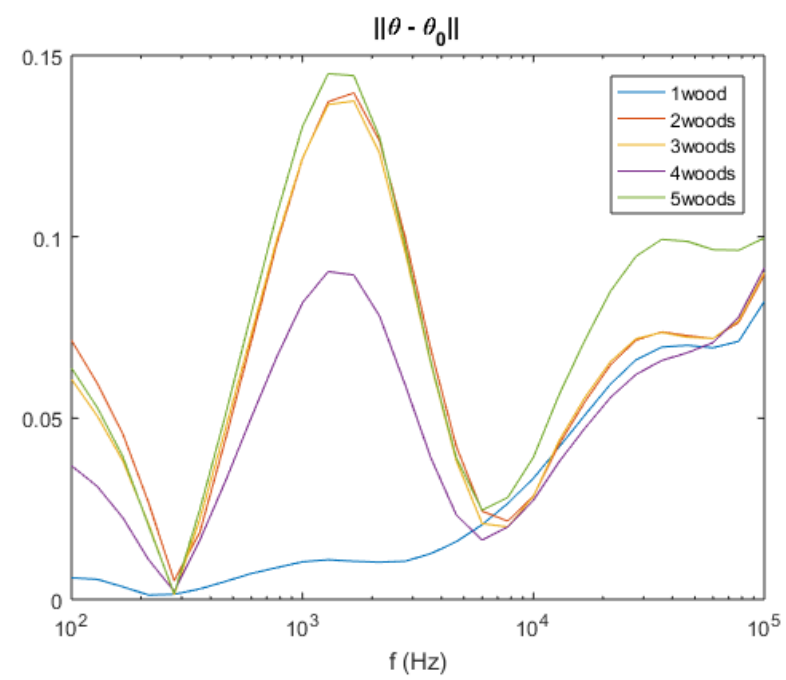

Fig. 2: Norm phase-difference for number of wood inclusions

A wood (balsa) occupies a single grid in the liquid metal (GaInSn) column. Five classes are studied: 1 wood (central grid), 2 woods (East-West), 3 woods (East-centre-West), 4 woods (North-South East-West), and 5 woods (North-South centre East-West), as shown in Fig. 3. Each case is measured in separate sessions and accompanied by the respective reference measurement.

Datasets are created and labelled for five classes, and split into training $(80 \%)$ and validation (20\%) data. They are listed in Table I.

TABLE I: 1D Dataset

\begin{tabular}{cccc}
\hline Label & Class & Train Data & Test Data \\
\hline 0 & 1wood & 40 & 10 \\
1 & 2wood & 40 & 10 \\
2 & 3wood & 40 & 10 \\
3 & 4wood & 40 & 10 \\
4 & 5wood & 40 & 10 \\
\hline
\end{tabular}

Fig. 4 shows the architecture. Keras model Sequential is constructed consisting three hidden dense layers, each of which has 128 neurons, with activation function ReLU. Vector of "logits" scores for each class are then converted into probability using Softmax function. Loss function Sparse Categorical Crossentropy takes a vector of logits and a "True" index and returns a scalar loss for each example. The model is compiled using optimiser ADAptive with Momentum (Adam), utilising "accuracy" metrics to measure the loss and the accuracy of the model. This model will then be fitted adjusting parameters to minimise the loss.

Total number of parameters (param) are 53893, all of which are trainable. Model summary is described in Table II.

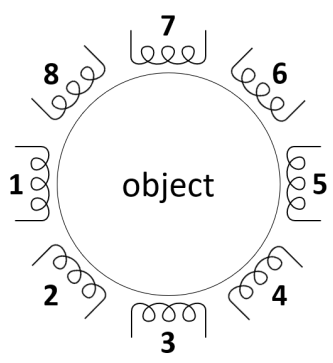

(a) 8-coil MIT sensor

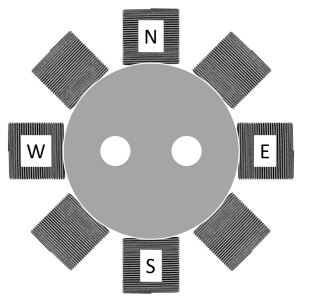

(c) case 1

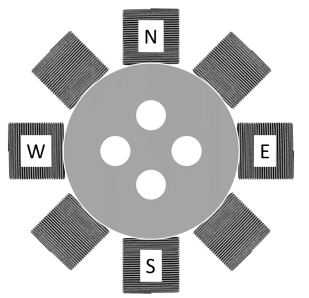

(e) case 3

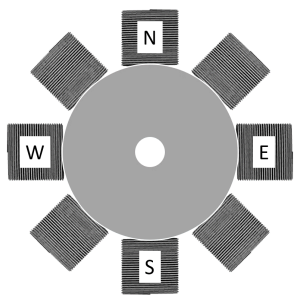

(b) $\operatorname{case} 0$

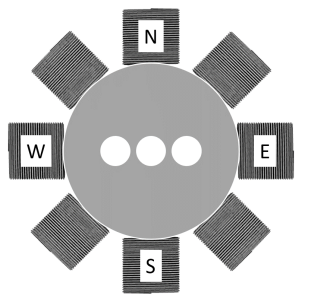

(d) case2

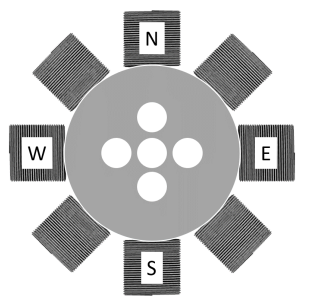

(f) case 4
Fig. 3: Wood inclusions in liquid metal cases

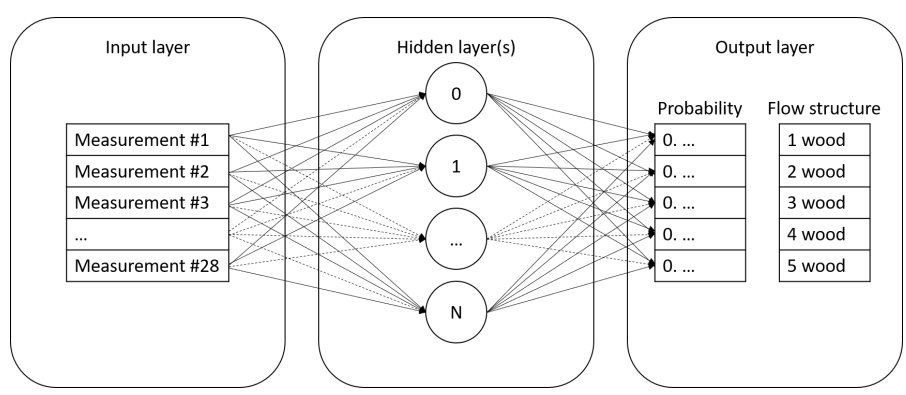

Fig. 4: A neural network with features, hidden layers, and predictions

TABLE II: FCNN Model Summary

\begin{tabular}{llll}
\hline Layer & Properties & Output Shape & Param \\
\hline input & $28 \times 1$ & $(28)$ & 0 \\
Dense1 & Activation: ReLU & $(128)$ & 3712 \\
Dense2 & Activation: ReLU & $(128)$ & 16512 \\
Dense3 & Activation: ReLU & $(128)$ & 16512 \\
Dense4 & Activation: ReLU & $(128)$ & 16512 \\
Dense5 & + Softmax & $(5)$ & 645 \\
\hline
\end{tabular}



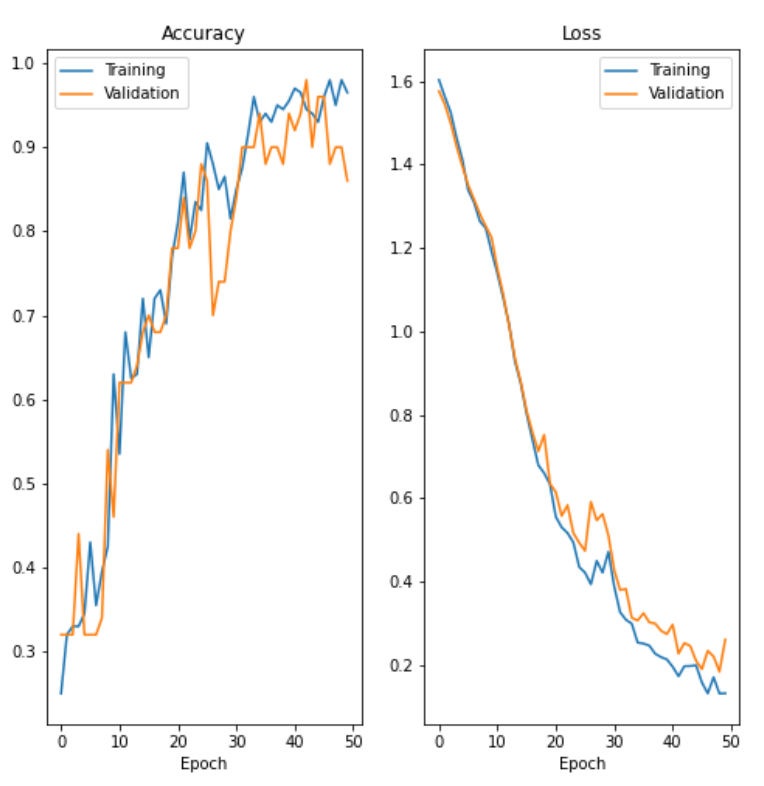

Fig. 5: FCNN training and validation accuracy-loss vs epoch

Fig. 5 shows training and validation accuracy-loss along 50 epochs. At the beginning ( $0 \mathrm{~s} 23 \mathrm{~ms} / \mathrm{step})$ train loss is 1.6027 , train accuracy is 0.2500 , validation loss is 1.5758 , and validation accuracy is 0.3200 ; at the end $(0 \mathrm{~s} 4 \mathrm{~ms} / \mathrm{step})$ train loss is 0.1324 , train accuracy is 0.9650 , validation loss is 0.2612 , and validation accuracy is 0.8600 . Performance is checked on validation/test set, giving: test loss 0.26 and test accuracy 0.86 . The accuracy on the validation dataset is going lesser than the accuracy on the training dataset starting at epoch $>40$, indicating overfitting.

Examples of prediction on woods/voids are depicted in Fig. 6. Ten test data are fed into the model, and prediction bar chart is shown accordingly.

Confusion matrix is shown in Fig. 7. The map represents tests, where each case has ten predictions. Sample with two woods inclusions has the highest "True" vs "Predicted" value; whereas three woods case is the least accurate. The classifier more likely produces errors because they are relatively similar patterns and close void locations.

\section{CNN CLASSIFIER}

Two-dimensional data in the form of multi-frequency phasedifference coil combination are constructed as pseudo-image. This makes 2D analysis approach is suitable, such as applying CNN model.

Mutual coil pairs measurements lie on the horizontal axis; whereas frequency points on the vertical axis. Mutual inductance combinations are 28 (from eight coils), so to shape the image into 2D form, the same number of frequency points are set to 28 . The measurement frequency is swept in logarithmic fashion from $100 \mathrm{~Hz}$ up to $100 \mathrm{kHz}$. An example of a pseudoimage is shown in Fig 8.

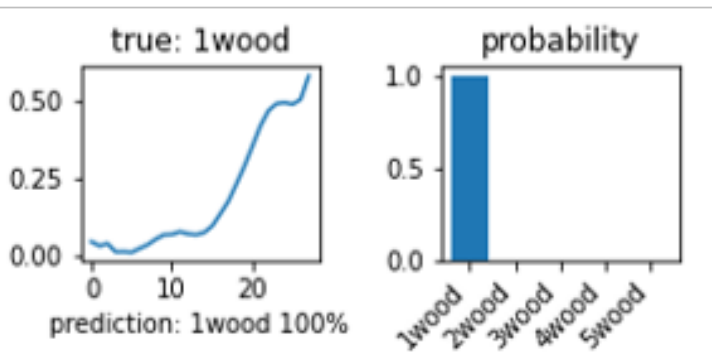

(a) one wood

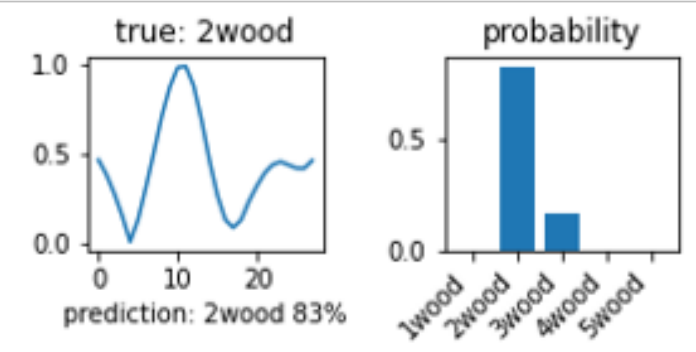

(b) two woods
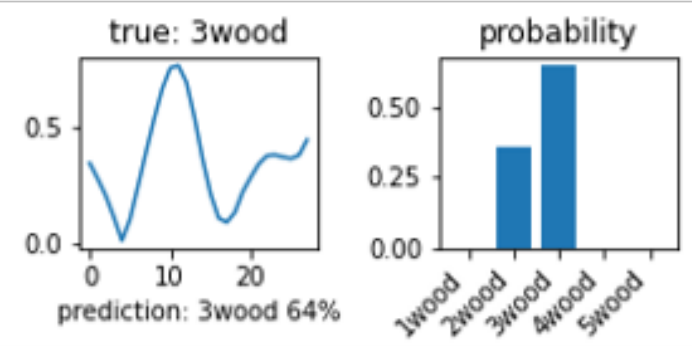

(c) three woods
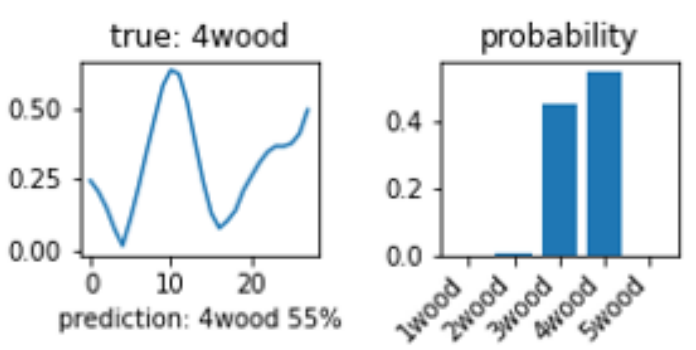

(d) four woods
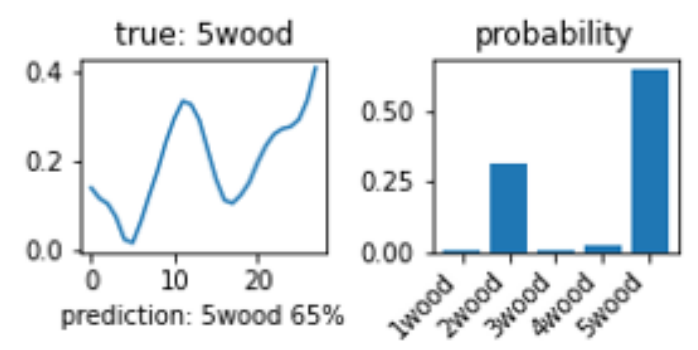

(e) five woods

Fig. 6: Plot prediction of sample case 


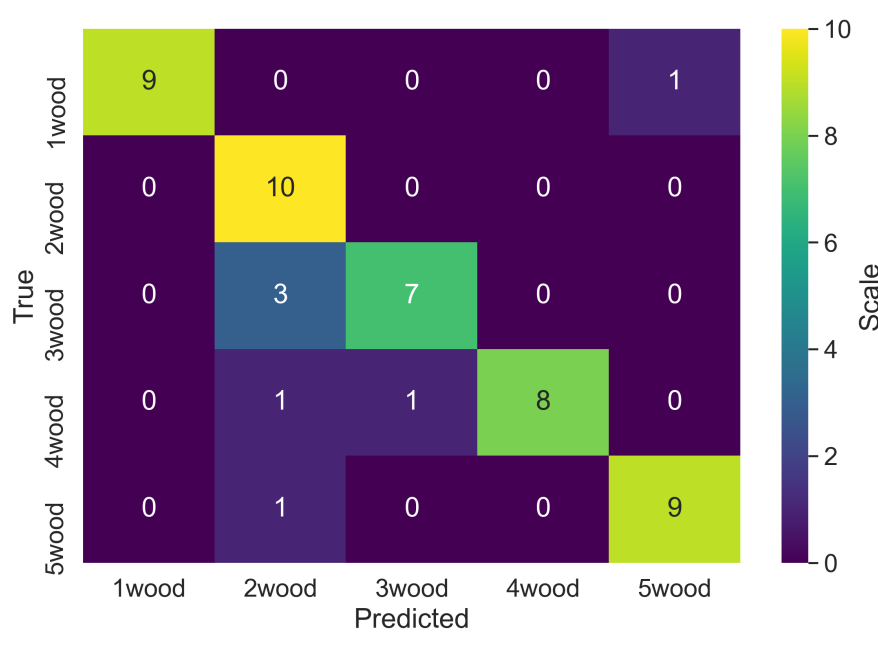

Fig. 7: FCNN confusion matrix

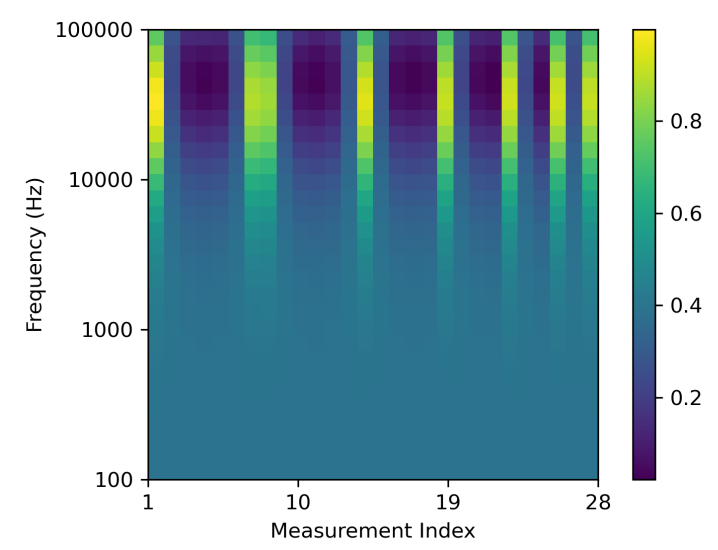

Fig. 8: Pseudo image multi-frequency mutual induction

The pseudo-image is $28 \times 28$ pixels and the values are scaled between $\left[\begin{array}{ll}0 & 1\end{array}\right]$. Each value represents phase-difference measurement of liquid metal case against free-space (air) background reference. In addition to cases depicted in Fig. 3, up to nine inclusions are given, and a full liquid metal (no inclusion) is incorporated. Therefore, there are ten classes to investigate, as listed in Table III.

Sequential layers consist of Conv2D, MaxPooling2D, and Dense are stacked for the model, as illustrated in Fig. 9. The diagram gives the information about input shape, which is a pseudo-image in 2D 28x28 pixels and one 'colour' channel. Subsequent layers are convolution and pooling, before the tensor is flattened for traditional dense neural network. Finally, the last layer provides a number of outputs according to the prediction classes. Table IV describes the architecture in detail, where total (trainable) parameters are 93322.

Fig. 10 shows training and validation accuracy-loss along 250 epochs. Performance is checked on validation/test set, giving: test loss 0.18 and test accuracy 0.96. Examples of prediction cases are depicted in Fig 11. Ten test data are fed into the model, and prediction bar chart is shown accordingly.
TABLE III: 2D Dataset

\begin{tabular}{cccc}
\hline Label & Class & Train Data & Test Data \\
\hline 0 & 0wood & 40 & 10 \\
1 & 1wood & 40 & 10 \\
2 & 2 wood & 40 & 10 \\
3 & 3 wood & 40 & 10 \\
4 & 4 wood & 40 & 10 \\
5 & 5 wood & 40 & 10 \\
6 & 6wood & 40 & 10 \\
7 & 7wood & 40 & 10 \\
8 & 8wood & 40 & 10 \\
9 & 9wood & 40 & 10 \\
\hline
\end{tabular}

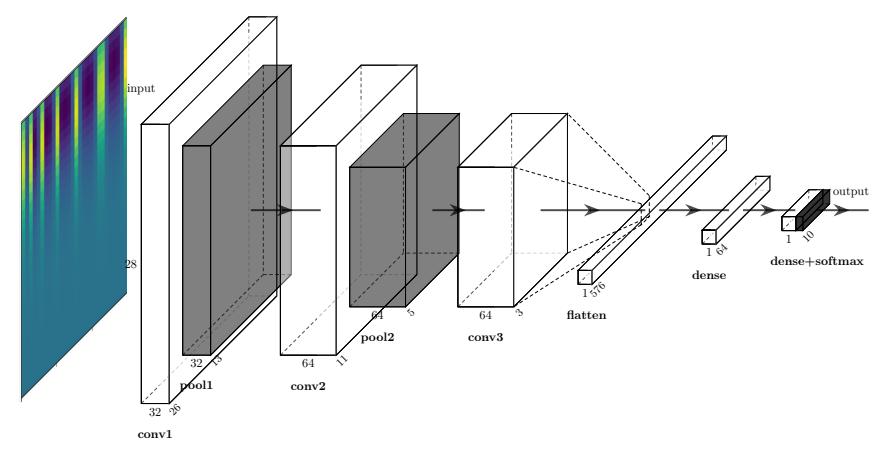

Fig. 9: CNN model

TABLE IV: CNN Model Summary

\begin{tabular}{|c|c|c|c|c|c|}
\hline Layer & Properties & Stride & Padding & Output Shape & Param \\
\hline input & $28 \times 28 \times 1$ & - & - & $(28,28,1)$ & 0 \\
\hline Convolution1 & $\begin{array}{l}\text { Filters: } 32 \\
\text { Kernel: } 3 \times 3 \\
\text { Activation: ReLU }\end{array}$ & $1 \times 1$ & Valid & $(26,26,32)$ & 320 \\
\hline MaxPooling1 & Kernel: 2 × 2 & - & Valid & $(13,13,32)$ & 0 \\
\hline Convolution2 & $\begin{array}{l}\text { Filters: } 64 \\
\text { Kernel: } 3 \text { × } 3 \\
\text { Activation: ReLU }\end{array}$ & $1 \times 1$ & Valid & $(11,11,64)$ & 18496 \\
\hline MaxPooling2 & Kernel: 2 × 2 & - & Valid & $(5,5,64)$ & 0 \\
\hline Convolution3 & $\begin{array}{l}\text { Filters: } 64 \\
\text { Kernel: } 3 \times 3 \\
\text { Activation: ReLU }\end{array}$ & $1 \times 1$ & Valid & $(3,3,64)$ & 36928 \\
\hline Flatten & - & - & - & $(576)$ & 0 \\
\hline Dense1 & Activation: ReLU & - & - & (64) & 36928 \\
\hline Dense2 & + Softmax & - & - & (10) & 650 \\
\hline
\end{tabular}
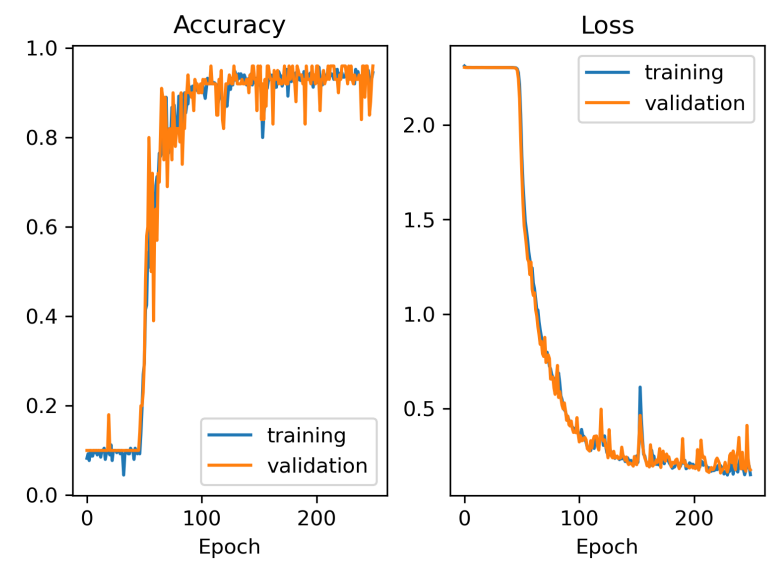

Fig. 10: CNN training and validation accuract-loss vs epoch 
(a) case 0

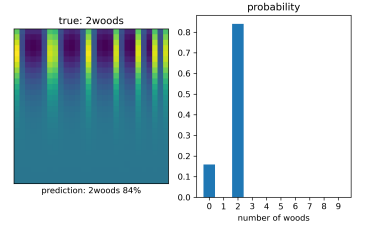

(c) case 2

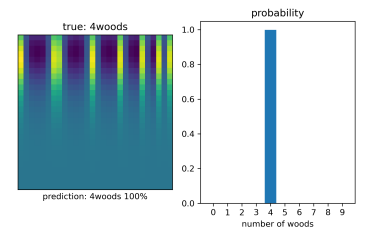

(e) case 4

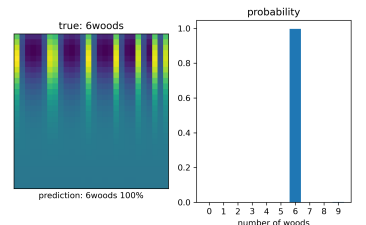

(g) case6

(i) case 8
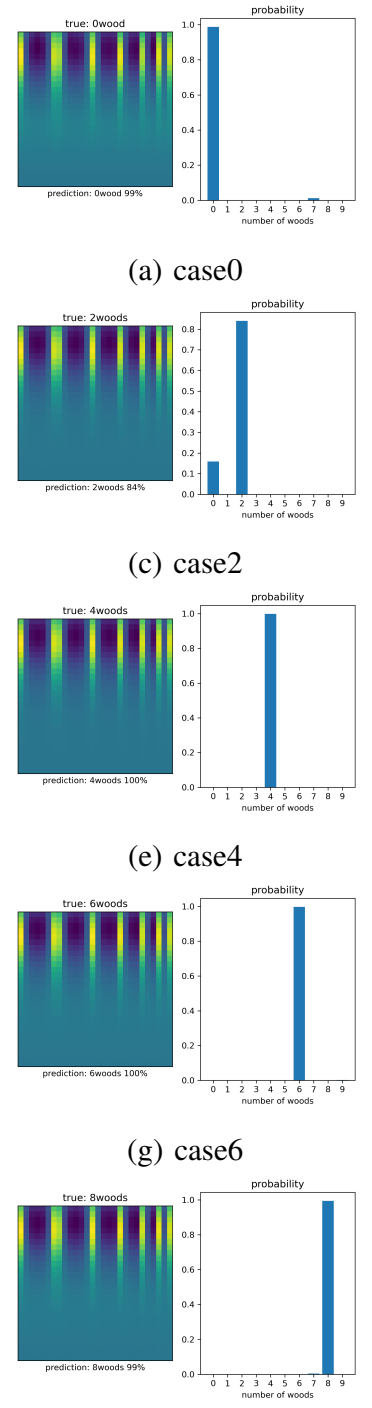

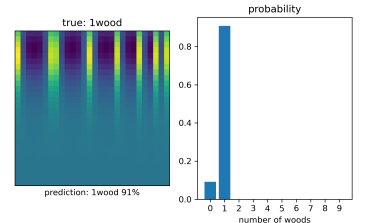

(b) case 1

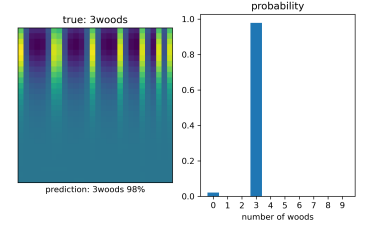

(d) case 3

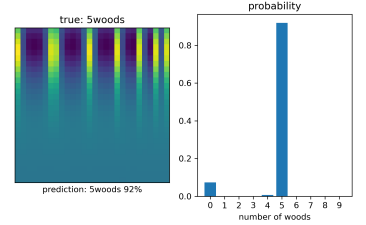

(f) case 5

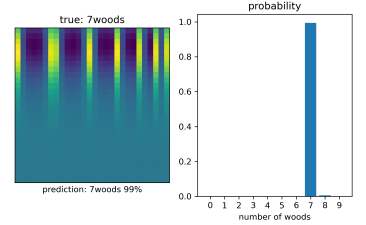

(h) case 7

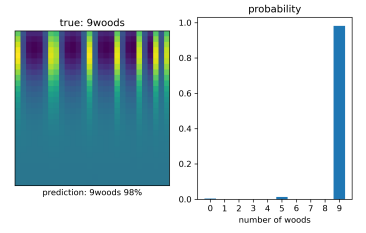

(j) case 9
Fig. 11: CNN prediction sample

Confusion matrix is mapped in Fig. 12. For clarity, the labels associated with inclusion numbers are presented on the axes. Almost all test samples are predicted accurately, except for full case (no inclusion) where the classifier mispredicts symmetrically distributed of one and five voids/woods (NMI).

\section{Discussion}

In this study, we define the classification based on the number of voids. It is possible to define the classification in different ways depending on the application. The accuracy of the classifier, on the one hand, depends on the algorithm and training strategies and the other hand depends on the accuracy of the MIT data. To prevent the overfitting, more training data are required, also, additional weight regularisation might be needed. With an interest in the interior region of the liquid metal, the accuracy of the MIT setup for low-frequency data will be an important factor.

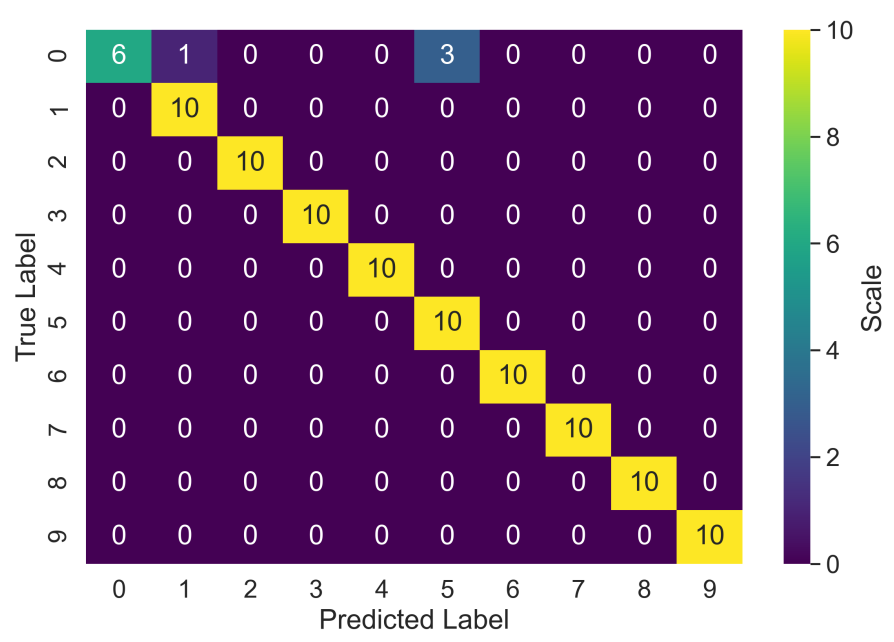

Fig. 12: CNN confusion matrix

\section{CONCLUSION}

The work proposes a liquid metal flow condition classifier focused on the interior voidage. Measurement datasets are multi-frequency mutual induction phase-difference of several wood inclusion variations inside liquid metal GaInSn. The 1D classifier architecture is a multi-layered fully connected neural network (FCNN). After 50 epochs, this model produces a training loss of 0.13 , training accuracy 0.97 ; whereas validation loss 0.26 , validation accuracy 0.86 . The $2 \mathrm{D}$ classifier architecture is based on a convolutional neural network (CNN). After 250 epochs, this model produces a training loss of 0.15 , training accuracy 0.95 ; whereas validation loss 0.18 , validation accuracy 0.96 . The number of woods, or non-metallic inclusions (NMI), classification can be further translated into other quantification such as interior void fraction percentage. This framework provides a prospect for a data-driven liquid metal processing system.

\section{APPENDIX I}

\section{SUPPLEMENTARY MATERIALS}

Dataset and script are available upon request: https://github.bath.ac.uk/im463/metal-flow

\section{ACKNOWLEDGMENT}

This research made use of the Balena High Performance Computing (HPC) Service at the University of Bath. The authors would like to thank Dr Thomas Wondrak (HZDR), Ir. Dirk van der Plas, Ir. Sjaak van Oord, and Dr. Daniel van Odyck (Tata Steel) for their suggestions on the experiment. David Chapman 's support in measurement setup is also acknowledged. Fig. 9 was visualised using modified version of PlotNeuralNet [31].

\section{REFERENCES}

[1] T. Wondrak, U. Hampel, M. Ratajczak, I. Glavinic, F. Stefani, S. Eckert, D. van der Plas, P. Pennerstorfer, I. Muttakin, M. Soleimani, S. Abouelazayem, J. Hlava, A. Blishchik, and S. Kenjeres, "Real-time control of the mould flow in a model of continuous casting in frame of the TOMOCON project," IOP Conference Series: Materials Science and Engineering, vol. 424, p. 012003, oct 2018. 
[2] J. Banhart, "Manufacturing routes for metallic foams," JOM, vol. 52, no. 12, pp. 22-27, Dec. 2000. [Online]. Available: http://link.springer.com/10.1007/s11837-000-0062-8

[3] C. Corazza, K. Rosseel, W. Leysen, K. Gladinez, A. Marino, J. Lim, and A. Aerts, "Optical fibre void fraction detection for liquid metal fast neutron reactors," Experimental Thermal and Fluid Science, vol. 113, p. 109865, 2020. [Online]. Available: https://www.sciencedirect.com/science/article/pii/S0894177719303681

[4] I. Muttakin and M. Soleimani, "Magnetic induction tomography spectroscopy for structural and functional characterization in metallic materials," Materials, vol. 13, no. 11, p. 2639, Jun 2020. [Online]. Available: http://dx.doi.org/10.3390/ma13112639

[5] K. Suzuki, "Overview of deep learning in medical imaging," Radiological Physics and Technology, vol. 10, no. 3, pp. 257-273, Sep. 2017. [Online]. Available: http://link.springer.com/10.1007/s12194-0170406-5

[6] Z. Zhang and E. Sejdić, "Radiological images and machine learning: Trends, perspectives, and prospects," Computers in Biology and Medicine, vol. 108, pp. 354-370, 2019. [Online]. Available: https://www.sciencedirect.com/science/article/pii/S0010482519300642

[7] G. Wang, "A perspective on deep imaging," IEEE Access, vol. 4, pp. 8914-8924, 2016.

[8] M. Araya-Polo, J. Jennings, A. Adler, and T. Dahlke, "Deep-learning tomography," The Leading Edge, vol. 37, no. 1, pp. 58-66, 2018. [Online]. Available: https://doi.org/10.1190/tle37010058.1

[9] G. Wang, J. C. Ye, and B. De Man, "Deep learning for tomographic image reconstruction," Nature Machine Intelligence, vol. 2, no. 12, pp. 737-748, Dec. 2020. [Online]. Available: http://www.nature.com/articles/s42256-020-00273-z

[10] Y. Wu, B. Chen, K. Liu, C. Zhu, H. Pan, J. Jia, H. Wu, and J. Yao, "Shape reconstruction with multiphase conductivity for electrical impedance tomography using improved convolutional neural network method," IEEE Sensors Journal, pp. 1-1, 2021.

[11] Z. Wei, D. Liu, and X. Chen, "Dominant-current deep learning scheme for electrical impedance tomography," IEEE Transactions on Biomedical Engineering, vol. 66, no. 9, pp. 2546-2555, Sep. 2019.

[12] Z. Chen, Q. Yuan, X. Song, C. Chen, D. Zhang, Y. Xiang, R. Liu, and Q. Xuan, "Mitnet: Gan enhanced magnetic induction tomography based on complex cnn," 2021.

[13] J. Yao, H. Chen, Z. Xu, J. Huang, J. Li, J. Jia, and H. Wu, "Development of a wearable electrical impedance tomographic sensor for gesture recognition with machine learning," IEEE Journal of Biomedical and Health Informatics, vol. 24, no. 6, pp. 1550-1556, June 2020.

[14] S. Kaji and S. Kida, "Overview of image-to-image translation by use of deep neural networks: denoising, super-resolution, modality conversion, and reconstruction in medical imaging," Radiological Physics and Technology, vol. 12, no. 3, pp. 235-248, Sep. 2019. [Online]. Available: http://link.springer.com/10.1007/s12194-019-00520-y

[15] H. Lee, C. Huang, S. Yune, S. H. Tajmir, M. Kim, and S. Do, "Machine Friendly Machine Learning: Interpretation of Computed Tomography Without Image Reconstruction," Scientific Reports, vol. 9, no. 1, p. 15540, Dec. 2019. [Online]. Available: http://www.nature.com/articles/s41598-019-51779-5

[16] O. Costilla-Reyes, P. Scully, and K. B. Ozanyan, "Deep neural networks for learning spatio-temporal features from tomography sensors," IEEE Transactions on Industrial Electronics, vol. 65, no. 1, pp. 645-653, Jan 2018.

[17] J. Li, D. Hu, W. Chen, Y. Li, M. Zhang, and L. Peng, "Cnn-based volume flow rate prediction of oil-gas-water three-phase intermittent flow from multiple sensors," Sensors, vol. 21, no. 4, 2021. [Online]. Available: https://www.mdpi.com/1424-8220/21/4/1245

[18] Q. Guo, M. Ye, W. Yang, and Z. Liu, "A machine learning approach for electrical capacitance tomography measurement of gas-solid fluidized beds," AIChE Journal, vol. 65, no. 6, p. e16583, 2019. [Online]. Available: https://aiche.onlinelibrary.wiley.com/doi/abs/10.1002/aic.16583

[19] I. Muttakin, T. Wondrak, and M. Soleimani, "Magnetic induction tomography sensors for quantitative visualization of liquid metal flow shape," IEEE Sensors Letters, vol. 4, no. 7, pp. 1-4, July 2020.

[20] I. Mohanty and D. Bhattacherjee, "Artificial Neural Network and Its Application in Steel Industry," in Computational Approaches to Materials Design: Theoretical and Practical Aspects, S. Datta and J. P. Davim, Eds. Hershey, PA: IGI Global, 2016, pp. 267-300. [Online]. Available: http://doi:10.4018/978-1-5225-0290-6.ch010

[21] C. Kusche, T. Reclik, M. Freund, T. Al-Samman, U. Kerzel, and S. Korte-Kerzel, "Large-area, high-resolution characterisation and classification of damage mechanisms in dual-phase steel using deep learning," PLOS ONE, vol. 14, no. 5, pp. 1-22, 05 2019. [Online]. Available: https://doi.org/10.1371/journal.pone.0216493

[22] H. Shin, H. R. Roth, M. Gao, L. Lu, Z. Xu, I. Nogues, J. Yao, D. Mollura, and R. M. Summers, "Deep convolutional neural networks for computer-aided detection: Cnn architectures, dataset characteristics and transfer learning," IEEE Transactions on Medical Imaging, vol. 35, no. 5, pp. 1285-1298, May 2016.

[23] N. Alqahtani, F. Alzubaidi, R. T. Armstrong, P. Swietojanski, and P. Mostaghimi, "Machine learning for predicting properties of porous media from $2 \mathrm{~d}$ x-ray images," Journal of Petroleum Science and Engineering, vol. 184, p. 106514, 2020. [Online]. Available: https://www.sciencedirect.com/science/article/pii/S0920410519309350

[24] I. Muttakin and M. Soleimani, "Noninvasive conductivity and temperature sensing using magnetic induction spectroscopy imaging," IEEE Transactions on Instrumentation and Measurement, vol. 70, pp. 1-11, 2021.

[25] S. P. Sotiroudis, P. Sarigiannidis, S. K. Goudos, and K. Siakavara, "Fusing diverse input modalities for path loss prediction: A deep learning approach," IEEE Access, vol. 9, pp. 30441-30 451, 2021.

[26] Y. Li, X. Qin, Z. Zhang, and H. Dong, "A robust identification method for nonferrous metal scraps based on deep learning and superpixel optimization," Waste Management \& Research, vol. 0, no. 0, p. 0734242X20987884, 2021, pMID: 33499775. [Online]. Available: https://doi.org/10.1177/0734242X20987884

[27] J. Zheng, J. Li, Y. Li, and L. Peng, "A benchmark dataset and deep learning-based image reconstruction for electrical capacitance tomography," Sensors, vol. 18, no. 11, 2018. [Online]. Available: https://www.mdpi.com/1424-8220/18/11/3701

[28] N. Vahabi, R. Yerworth, M. Miedema, A. van Kaam, R. Bayford, and A. Demosthenous, "Deep analysis of eit dataset to classify apnea and non-apnea cases in neonatal patients," IEEE Access, vol. 9, pp. $25131-$ 25139,2021

[29] F. Chollet et al., "Keras," https://keras.io, 2015.

[30] M. Abadi, A. Agarwal, P. Barham, E. Brevdo, Z. Chen, C. Citro, G. S. Corrado, A. Davis, J. Dean, M. Devin, S. Ghemawat, I. Goodfellow, A. Harp, G. Irving, M. Isard, Y. Jia, R. Jozefowicz, L. Kaiser, M. Kudlur, J. Levenberg, D. Mané, R. Monga, S. Moore, D. Murray, C. Olah, M. Schuster, J. Shlens, B. Steiner, I. Sutskever, K. Talwar, P. Tucker, V. Vanhoucke, V. Vasudevan, F. Viégas, O. Vinyals, P. Warden, M. Wattenberg, M. Wicke, Y. Yu, and X. Zheng, "TensorFlow: Large-scale machine learning on heterogeneous systems," 2015, software available from tensorflow.org. [Online]. Available: https://www.tensorflow.org/

[31] H. Iqbal, "Harisiqbal88/plotneuralnet v1.0.0," Dec. 2018. [Online]. Available: https://doi.org/10.5281/zenodo.2526396 